\title{
The Absorption Factor in Crystal Spectroscopy
}

Cite as: Review of Scientific Instruments 10, 221 (1939); https://doi.org/10.1063/1.1751537 Submitted: 11 April 1939 . Published Online: 27 December 2004

Gustav Albrecht
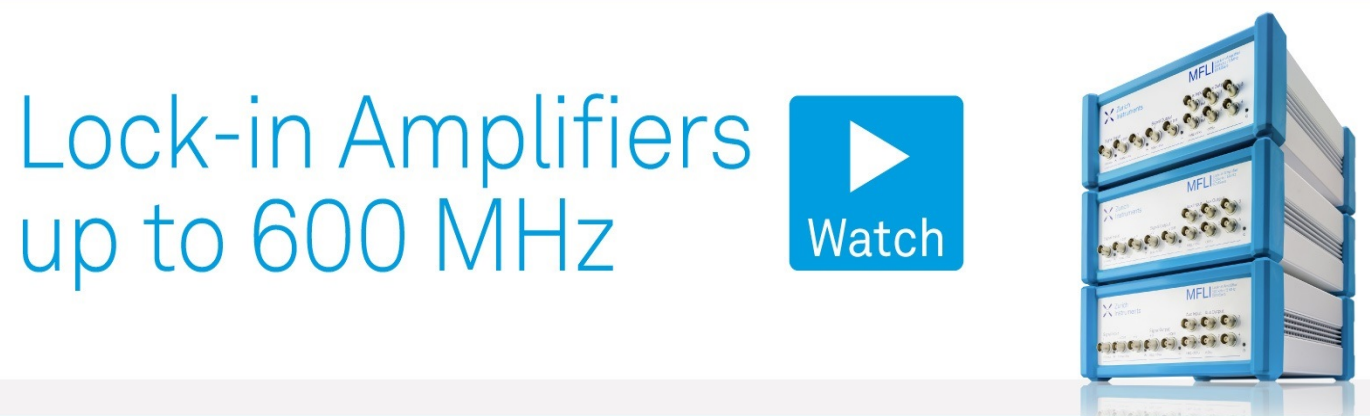


\section{Contributed Articles}

\section{The Absorption Factor in Crystal Spectroscopy*

\author{
Gustav Albrecht \\ California Institute of Technology, Pasadena, California
}

(Received April 11, 1939)

$I^{\mathrm{N}}$ $\mathrm{N}$ the determination of the structure of a crystal with x-rays, it is essential that a reliable set of diffraction intensity data be obtained. During its passage through the crystal, the $\mathrm{x}$-ray beam suffers a diminution in intensity due to absorption by the crystal itself, and the extent of this absorption will in general be different for different crystal planes. This variable factor has long been troublesome in crystal work, since its estimation presents a difficult problem even in simple special cases. The case of a cylindrical powder sample has been treated by various investigators, ${ }^{1-3}$ notably $\mathrm{A}$. Claassen ${ }^{4}$ and A. J. Bradley. ${ }^{5}$ The latter also treats the case of equatorial reflections from a single crystal ground to a cylinder. O. P. Hendershot ${ }^{6}$ has recently published a method applicable to a crystal of any shape but restricted to crystals of high absorbing power. Absorption effects in Weissenberg photographs have been treated by A. F. Wells ${ }^{7}$ and M. J. Buerger. ${ }^{8}$ In all these methods the computational labor is great, and the general case has not, to the author's knowledge, been treated. In view of these difficulties the usual procedure is to use a crystal so small as to make absorption negligible (necessitating long exposure) or to hold this unknown factor responsible for poor intensity agreement. A graphical method for treating absorption in a crystal of any size, shape and absorbing power, which has proved useful in the author's work, is here presented.

* Contribution from the Gates and Crellin Laboratories of Chemistry, California Institute of Technology, No. 707.

1 G. Greenwood, Phil. Mag. 3, 963 (1927).

${ }^{2}$ H. Möller and A. Reis, Zeits. f. Physik. Chemie A139, 425 (1928).

3 A. Rusterholz, Helv. Phys. Acta 4, 68 (1931).

4 A. Claassen, Phil. Mag. 9, 57 (1930).

${ }^{5}$ A. J. Bradley, Proc. Phys. Soc. London 47, 879 (1935).

${ }^{6}$ Otis P. Hendershot, Rev. Sci. Inst. 8, 324 (1937).

7 A. F. Wells, Zeits. f. Krist. 96, 451-3 (1937)

${ }^{8}$ M. J. Buerger, Zeits. f. Krist. 99, 189-204 (1938).
If the intensity of an incident beam of x-rays is denoted by $I_{1}$, and the path length of the rays in the crystal by $x$, the intensity of the emergent beam, $I_{2}$, is given by

$$
I_{2}=I_{1} e^{-\mu x},
$$

where $\mu$ is the linear absorption coefficient for the substance composing the crystal. Since in an actual crystal the path length $x$ is a variable depending on the size and shape of the crystal and on the diffracting plane, the diffracted beam will possess an intensity given by

$$
I=I_{0} \int_{V} e^{-\mu x} d v / V
$$

where $I_{0}$ is the intensity which the diffracted beam would have possessed had it not been for absorption. This integral is difficult to evaluate but an approximate solution can be obtained graphically by dividing the crystal into small volume elements and summing the absorption effects of each. Eq. (2) may be written

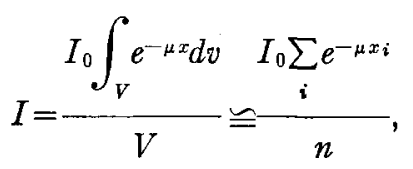

where $n$ volume elements are considered. In Fig. 1 the absorption by a volume element is given by

$$
I / I_{0}=e^{-\mu(a+b)},
$$

where $a+b$ is the path length in the crystal of the beam diffracted from this volume element. If the incident beam $S_{0}$ is divided into a number of equal rays as shown in Fig. 2, where $\theta$ is the Bragg angle for the plane hol here considered, then $S$ will represent the diffracted beam, divided into rays of the same dimensions as $S_{0}$. If the 
projection of a volume element is represented by the intersection of two ray lines, it is possible to obtain a value for the summation of Eq. (3) by adding quantities of the type $e^{-\mu x_{i}}$ and dividing this sum by $n$, the number of ray intersections associated with the diagram. The path length for a given volume element can be measured with rulers and the quantity $e^{-\mu x_{i}}$ can be readily obtained from an exponential table. It is thus only necessary to draw a cross section of the actual crystal to scale; draw in the incident and emergent rays in the direction appropriate to the plane under consideration; measure the path lengths, and count their number. If a large number of calculations are to be performed the work may be facilitated by constructing two masks, consisting of parallel ruled lines on celluloid to be layed over the drawing of the crystal section instead of drawing in the rays for each plane calculated.

It was assumed in the above that the entire

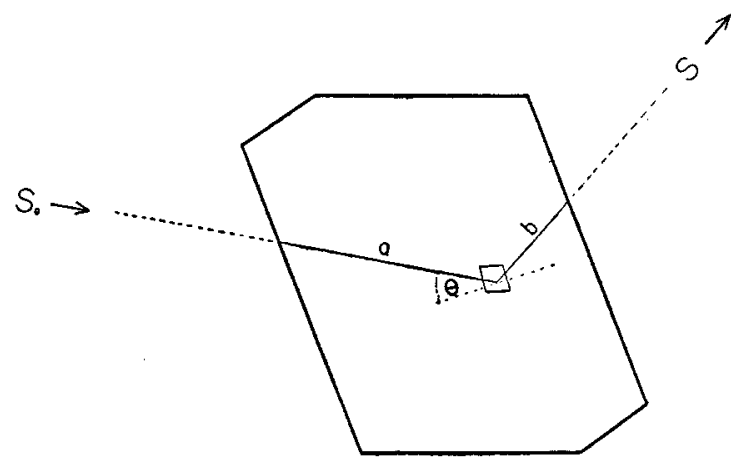

FIG. 1.

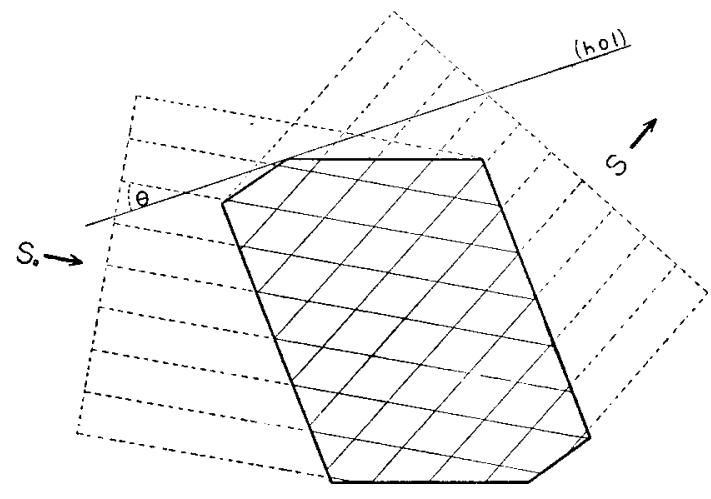

FIG. 2.

crystal was bathed in the x-ray beam, but the method can be readily extended to the case of a crystal larger than the beam, providing that the cross section of the beam and the portion of the crystal upon which it impinges are known. If the cross section of the crystal perpendicular to the section considered is not constant, as is often the case, for example in crystals with pyramidal ends, it is necessary to repeat the above process for a number of layers to obtain a good value of the absorption. It should be pointed out that any desired degree of approximation can be obtained by increasing the number of rays used in the diagrams, but surprisingly good results are obtainable using a rather small number of rays. It is obvious that the shape of the crystal presents no difficulties in this method. Except for the effect of secondary extinction, this procedure approximates the mechanism of absorption which actually takes place in the crystal. 